\title{
Association between vestibular schwannomas and mobile phone use
}

\author{
In Seok Moon • Bo Gyung Kim • Jinna Kim • \\ Jong Dae Lee • Won-Sang Lee
}

Received: 9 July 2013 / Accepted: 5 August 2013 / Published online: 27 August 2013

(C) The Author(s) 2013. This article is published with open access at Springerlink.com

\begin{abstract}
Vestibular schwannomas (VSs) grow in the region where the energy from mobile phone use is absorbed. We examined the associations of VSs with mobile phone use. This study included 119 patients who had undergone surgical tumor removal. We used two approaches in this investigation. First, a case-control study for the association of mobile phone use and incidence of VSs was conducted. Both cases and controls were investigated with questions based on INTERPHONE guidelines. Amount of mobile phone use according to duration, daily amount, and cumulative hours were compared between two groups. We also conducted a case-case study. The location and volume of the tumors were investigated by MRI. Associations between the estimated amount of mobile phone use and tumor volume and between the laterality of phone use and tumor location were analyzed. In a case-control study, the odds ratio (OR) of tumor incidence according to mobile phone use was 0.956 . In the case-case study, tumor volume and estimated cumulative hours showed a strong correlation $\left(r^{2}=0.144, p=0.002\right)$, and regular mobile phone users showed tumors of a markedly larger volume than those of non-regular users $(p<0.001)$. When the analysis was limited to regular users
\end{abstract}

Electronic supplementary material The online version of this article (doi:10.1007/s13277-013-1081-8) contains supplementary material, which is available to authorized users.

I. S. Moon • B. G. Kim • W.-S. Lee $(\bowtie)$

Department of Otorhinolaryngology, Yonsei University College

of Medicine, 134 Sinchon-dongSeodaemun-gu Seoul 120-752,

South Korea

e-mail: ismoonmd@yuhs.ac

J. Kim

Department of Radiology, Yonsei University College of Medicine, Seoul, South Korea

J. D. Lee

Department of Otorhinolaryngology-Head and Neck Surgery,

Soonchunhyang University College of Medicine,

Bucheon, South Korea who had serviceable hearing, laterality showed a strong correlation with tumor side $(\mathrm{OR}=4.5)$. We found that tumors may coincide with the more frequently used ear of mobile phones and tumor volume that showed strong correlation with amount of mobile phone use, thus there is a possibility that mobile phone use may affect tumor growth.

Keywords Vestibular schwannoma $\cdot$ Mobile phone · Electromagnetic fields

\section{Introduction}

The awareness of vestibular schwannoma has increased over the past few decades [1]. Better diagnostic tools are likely responsible for a higher rate of diagnosis [2], but a number of environmental factors, including electromagnetic fields (EMFs) emitted by mobile phones, have been also suspected as potential risk factors for vestibular schwannoma [3].

The rapid increase in mobile phone use during the last decade has raised some safety concerns. In particular, a risk for vestibular schwannoma is associated with mobile phone use because the acoustic nerve tissue is a sound perception organ and is located close to where people hold their mobile phones during use.

There is some controversy surrounding the effect of the energy from mobile phones use on vestibular schwannoma. Several epidemiological studies have found no increase in the risk of vestibular schwannoma associated with mobile phone use [4-7]. These results are supported by studies that found that the EMFs produced from mobile phones do not have enough energy to break chemical bonds or damage DNA $[8,9]$. Other studies, however, have identified an increased risk of tumors in the more frequently used ear $[10,11]$. These results are supported by studies that reported that EMFs emitted from mobile phones can penetrate $4-5 \mathrm{~cm}$ into the brain, which can potentially increase the temperature of the tissue by up to $0.1{ }^{\circ} \mathrm{C}$. This thermal effect could consequently influence protein 
phosphorylation [12-14]. The possibility and mechanism of carcinogenicity was also reviewed in many studies including oxidative stress, apoptosis, and effects on immune function [15-18].

The case-control design is widely accepted as one of the most useful methods to analyze the relationship between mobile phone use and vestibular schwannoma. However, these studies are known to be vulnerable to selection and recall biases $[5,19]$. The case-case study design is also vulnerable to selection and recall biases, but the situation is less complicated than in case-control studies [20].

In previous case-case studies, the laterality of mobile phone use coinciding with the occurrence of vestibular schwannoma is often presented as evidence for association [21, 22]. Inskip et al. [21] suggested the following three assumptions: there is no risk from a mobile phone on the contralateral side, risk to the ipsilateral side is the same for left- and right-sided tumors, and the incidence of left- and right-sided tumors is the same for nonusers of mobile phones.

Most previous reports have diagnosed vestibular schwannoma through imaging, but even though schwannoma has characteristic image findings, a bias may arise since its pathology cannot be confirmed. In addition, previous reports that evaluated the coincidence between the laterality of mobile phone use and tumor side did not consider the patient's hearing. Unlike other tumors, vestibular schwannoma has a direct effect on the auditory system. Therefore, a bias may arise if the patient's hearing is not considered and only the coincidence between the laterality of mobile phone use and tumor side is evaluated. Finally, previous reports analyzed the association between EMF emitted by mobile phones and only incidence of tumors. However, if the main mechanism involves protein phosphorylation changes due to a heating effect rather than actual degeneration of DNA, then EMF might have the potential to increase/ decrease the growth of an existing tumor or change its shape even though it may not actually induce tumor development. Thus, an analysis of the effects of mobile phone use on tumor growth, rather than only tumor incidence, is required.

For this study, we recruited patients confirmed to have vestibular schwannoma after surgery, and the coincidence between the laterality of mobile phone use and tumor side was analyzed with consideration of patients' hearing. Finally, based on the hypothesis that "mobile phone use may affect the physiognomy of the vestibular schwannoma," we examined the associations between vestibular schwannomas and mobile phone use not only for the incidence of tumor but also for any differences in the growth or characteristics of tumors.

\section{Methods}

We used two approaches in this study: a case-control study and a case-case analysis. First, a case-control study for the relative risk of mobile phone use was conducted. We recruited 207 patients who underwent surgery and were pathologically confirmed to have vestibular schwannoma by the Department of Otorhinolaryngology at Severance Hospital, Seoul, South Korea, between January 1991 and December 2010. We were able to reach 134 of the 207 patients, and 119 of them agreed to reply to a questionnaire ( $88.8 \%$ participation rate) and were included in the case group. All patients were interviewed by telephone using the same questions. The questions were modified by authors based on INTERPHONE guidelines [23] by one interviewer from January to March 2012. The reference dates were set around the diagnosis date, and the questionnaire included the following: subject's history of mobile phone use, the year they began using a mobile phone, average daily number of outgoing and incoming calls, average call duration, dominant hand, proportion of calls using the left and right ears, and frequency of hands-free device use (Supplement 1). Age, gender, chief complaint at the first visit, past medical history, date of diagnosis (used as the reference date), tumor location (left or right), tumor volume, preoperative hearing threshold, and operative method were obtained as basic background information. We matched 238 controls (1:2 matching) who received comprehensive routine medical checkups (including brain MRI) in the authors' hospital for age, gender, and general health condition. The control subjects were also interviewed using the same questionnaire and matched the reference date. No controls had unilateral hearing loss.

A regular mobile phone user was defined as someone who had used a mobile phone at least once a week for the past 6 months. The average daily amount of mobile phone use was calculated by multiplying the average number of calls per day by the average talk time of one call. Cumulative hours of mobile phone use were calculated by multiplying the average daily amount of mobile phone use by the duration of mobile phone use. We compared the two groups in terms of duration of mobile phone use, daily mobile phone time, and cumulative hours of mobile phone use and calculated the relative risk of tumor incidence due to mobile phone use.

We also conducted a case-case study. The location and volume of the tumors were analyzed using imaging and a threedimensional volume calculation program (Aquaria INtuition ${ }^{\mathrm{TM}}$, TeraRecon, Foster City, CA) (Fig. 1). Mobile phone usage patterns were investigated with a questionnaire in the same manner as the case-control study. The duration, daily amount, and total cumulative hours were taken into account in determining cell phone usage. Subjects who had used a mobile phone for more than 10 years were classified as long-term users, those who used a mobile phone for more than $20 \mathrm{~min}$ per day were deemed heavy daily users, and cumulative heavy users were those who had used a mobile phone for more than 2,000 cumulative hours in their lifetimes. The cup points were set based on the references from previous reports. For each case, the volume of the tumor was compared according to each criterion. 
Fig. 1 Tumor volume was calculated using a 3D volume calculation program (Aquaria Intuition). Serial images of axial (a), coronal (b), and sagittal (c) cuts of gadolinium-enhanced temporal MRI were input into the program; (d) the tumor was reconstructed threedimensionally, and the tumor volume was automatically calculated

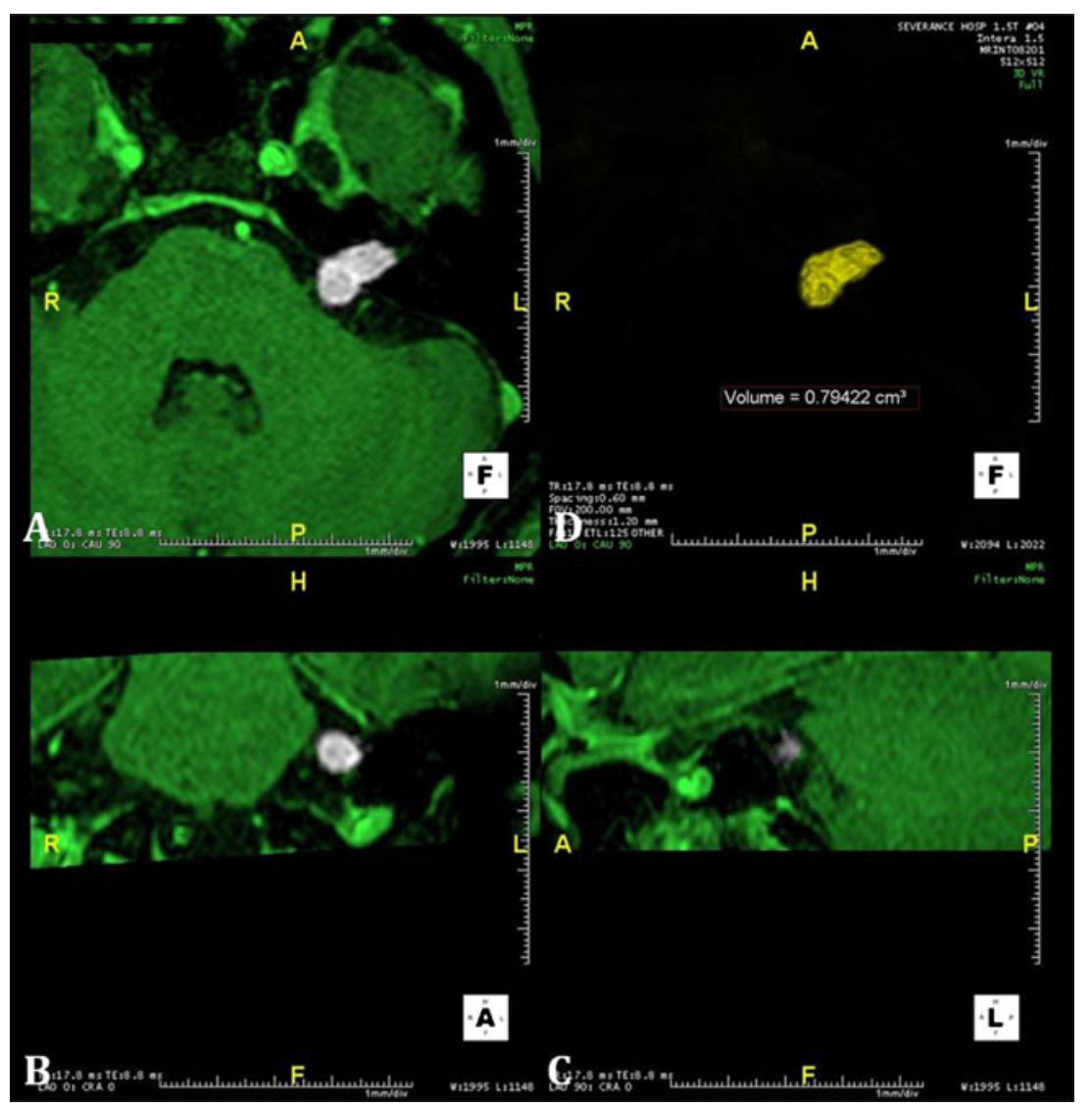

The correlation between the laterality of mobile phone use and tumor location (side) was also analyzed. The most frequently used ear was defined as the side used for more than three-quarters of the time spent on the phone. Subjects who did not meet this criterion were classified as having no dominant ear [4, 21]. If the tumor and the most frequently used ear were on the same side, the relationship was classified as ipsilateral; when the tumor and the most frequently used ear were on opposite side, the relationship was classified as contralateral. The risk ratio of vestibular schwannoma for mobile phone use was analyzed using multinominal logistic regression [21].

The study protocol was approved by the ethical committee of Yonsei University College of Medicine (no. 4-2012-0361), and consents were obtained from all participants.

\section{Results}

Case-control analysis

The basic characteristics of the cases and controls at the reference date of each group are shown in Table 1. There were no differences between the cases and controls in terms of age, gender, residential area, general health condition which may affect patients' immunity, and handedness $(p>0.05)$.

In the patient group, the longest delay from the date of diagnosis to the date of interview was 20 years. The dataset for analysis at the reference date showed that the most frequent tumor-related symptoms were tinnitus. Among these 119 cases, $64(53.8 \%)$ were mobile phone users at the reference date. With regard to dominant hand preference, 110 cases
Table 1 A case-control comparison of the basic characteristics of reference date

* Systemic disease means chronic debilitation disease which can affect patients' immunity such as uncontrolled DM, ESRD, and etc $\dagger$ Chi-square test or \$ Fischer's exact test for calculation of $p$ value

\begin{tabular}{llll}
\hline & Case $(n=119)$ & Control $(n=238)$ & $p$ value \\
\hline Age (years) & $47.92 \pm 13.14$ & $46.79 \pm 12.46$ & 0.543 \\
Gender (male/female) & $37: 82$ & $75: 161$ & $1.000^{\dagger}$ \\
Residential area (urban/rural) & $90: 29$ & $214: 24$ & $0.130^{\dagger}$ \\
\% of systemic disease & 18.4 & 8.8 & $0.077^{\ddagger}$ \\
\% of smoking & 7.3 & 8.8 & $0.812^{*}$ \\
Same handedness of mobile phone use (\%) & 42.8 & 56.7 & $0.084^{*}$ \\
\hline
\end{tabular}


Table 2 A case-control comparison of the amount of mobile phone use

\begin{tabular}{lcccl}
\hline & Case $(n=119)$ & Control $(n=238)$ & Odds ratio $(95 \% \mathrm{CI})$ & $p$ value \\
\hline Duration (years) & $10.15 \pm 5.39$ & $10.95 \pm 4.57$ & $0.961(0.910-1.012)$ & 0.201 \\
Time (min/day) & $25.78 \pm 40.76$ & $33.58 \pm 44.03$ & $0.998(0.945-1.041)$ & 0.43 \\
Cumulative hours (hrs) & $1778.61 \pm 2496.44$ & $2236.11 \pm 2533.02$ & $0.956(0.906-1.009)$ & 0.245 \\
\hline
\end{tabular}

(92.4\%) were right-handed and $4(3.4 \%)$ were left-handed, while $5(4.2 \%)$ were ambidextrous. In control participants, $201(84.5 \%)$ participants used mobile phone. Eleven (4.6\%) were left-handed and four $(1.7 \%)$ were ambidextrous.

Differences in the use of mobile phones between the patient and control groups are listed in Table 2. There were no statistically significant differences between the two groups $(p=0.245)$; in contrast, the control group had used cell phones a little bit longer than the patient group. The odds ratios of vestibular schwannoma incidence according to each parameter ranged from 0.956 to 0.998 . The reference date for the patient group was the date of diagnosis. For control group, the reference date was matched date to that of patient group.

\section{Case-case analysis}

Among the schwannoma group, there were no substantial differences between the regular and the non-regular phone users (Table 3 ). The average tumor volume of regular users ( $n=64)$ was $8.10 \pm 10.71 \mathrm{~cm}^{3}$, while non-regular users $(n=55)$ had a mean volume of $2.71 \pm 3.78 \mathrm{~cm}^{3}$. The difference in tumor volume between the two groups was statistically significant ( $p=0.004$, Fig. 2). The OR of regular user is 1.125 (CI 1.041-1.216, $p=0.003$ ).

When we limited our analysis to regular users, there was no significant difference $(p=0.130)$ in tumor size between longterm users $\left(9.83 \pm 11.97 \mathrm{~cm}^{3}, n=38\right)$ and short-term users $\left(5.57 \pm 8.15 \mathrm{~cm}^{3}, n=26\right)$, but a significant difference was observed between heavy users $\left(11.32 \pm 15.43 \mathrm{~cm}^{3}, n=32\right)$ and light users $\left(4.88 \pm 5.60 \mathrm{~cm}^{3}, n=32\right)$ based on daily amount of mobile phone use $(p=0.026)$. In terms of cumulative hours, tumor volume showed a strong correlation $\left(r^{2}=0.144\right.$, $p=0.002$ ) and was significantly larger in heavy users $\left(13.31 \pm 14.07 \mathrm{~cm}^{3}, n=26\right)$ than in light users $(4.88 \pm$ $\left.6.16 \mathrm{~cm}^{3}, n=38\right)(p=0.007)$ (Table 4 and Supplement 2).

Of the 64 regular mobile phone users, 12 cases (9\%) answered that they used both their left and right ears almost equally, while the others had laterality. Excluding these 12 cases, 52 cases were used for our risk analysis. Left-eardominant users $(31 / 52,59.6 \%)$ outnumbered right-eardominant users $(21 / 52,40.4 \%)$, but the difference was not significant $(p=0.592)$. Twenty-three cases were ipsilateral, and 29 were contralateral $(\mathrm{OR}=0.733)$. Since hearing is affected by tumor development and growth, the risk was reevaluated for patients who had serviceable hearing. Of the 21 patients with hearing better than $30 \mathrm{~dB}$ of the pure tone threshold and greater than $70 \%$ of speech recognition score, 12 cases were ipsilateral, 6 were contralateral, and 3 were both, and significant increase in risk was found $(\mathrm{OR}=4.50)$ (Table 5). Non-regular users who did not have laterality of mobile phone use showed similar tumor incidence on right and left side (Table 3).

\section{Discussion}

A case-control study was conducted under the hypothesis that the patient group used mobile phones more frequently. However, there was no difference between two groups in mobile phone use. There is a possibility of recall bias that the reference date for the case group was use of a mobile phone prior to surgery and matched day for the control group. The members of the case group would have a more concrete memory before and after the big event, the surgery, than that of the control group before and after the matched date. The ratio of mobile phone use in the control group could have been overestimated compared to the case group, in which would
Table 3 A case-case comparison of the basic characteristics of reference date

*Systemic disease means chronic debilitation disease which can affect patients' immunity such as uncontrolled DM, ESRD, and etc

$\dagger$ Chi-square test or $\ddagger$ Fischer's exact test for calculation of $p$ value

\begin{tabular}{llll}
\hline & Regular user $(n=64)$ & Non-regular user $(n=55)$ & $p$ value \\
\hline Age (years) & $46.80 \pm 11.74$ & $49.21 \pm 14.59$ & 0.321 \\
Gender (male/female) & $17: 47$ & $20: 35$ & $0.249^{\dagger}$ \\
Side of tumor (right/left) & $37: 27$ & $25: 30$ & $0.178^{\dagger}$ \\
Residential area (urban/rural) & $46: 16$ & $44: 13$ & $0.910^{\dagger}$ \\
\% of systemic disease* & 7.8 & 9.1 & $0.802^{\ddagger}$ \\
\% of smoking & 9.4 & 7.5 & $0.680^{\ddagger}$ \\
\hline
\end{tabular}




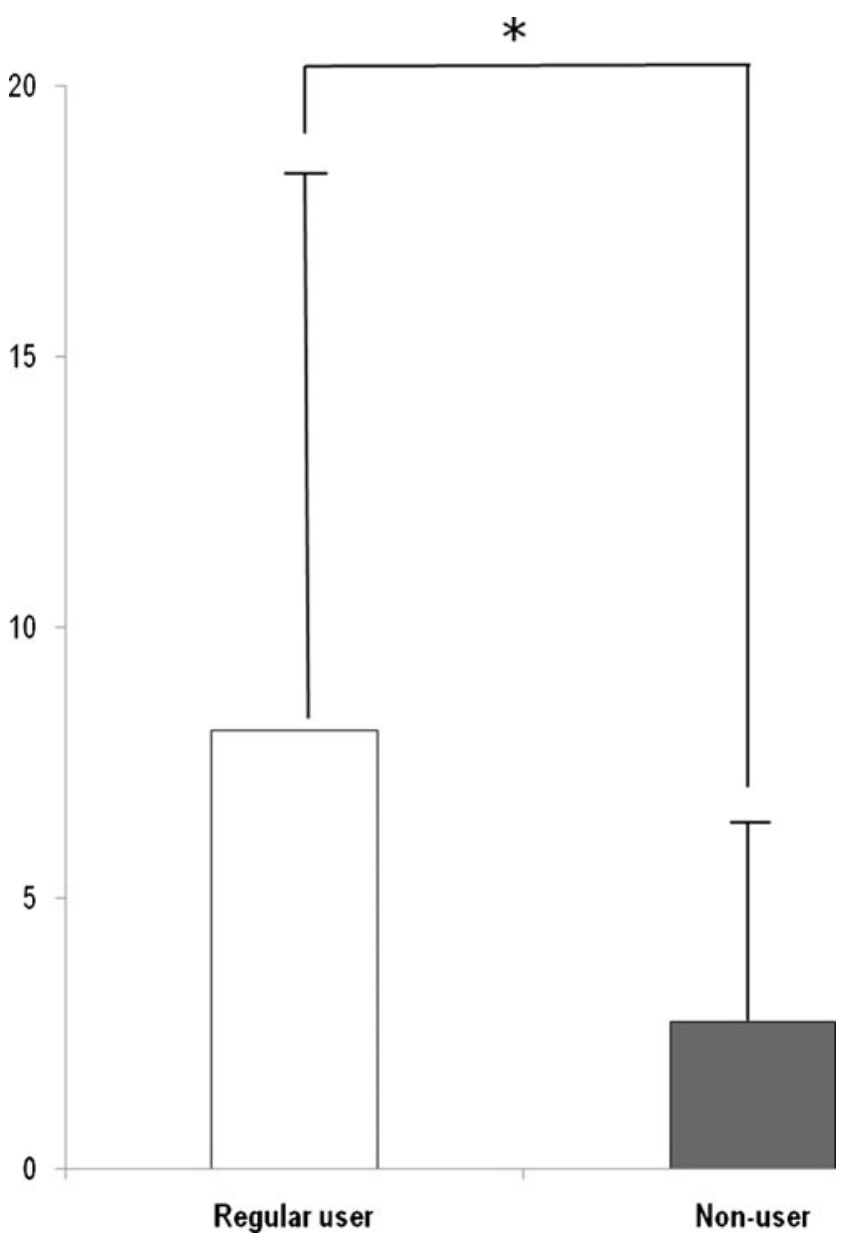

Fig. 2 The average tumor volume of regular mobile phone users $(8.10 \pm$ $\left.10.71 \mathrm{~cm}^{3}\right)$ was significantly larger than that of non-regular users $(2.71 \pm$ $\left.3.78 \mathrm{~cm}^{3}\right)(p<0.001)$. OR of regular user is 1.125 (CI 1.041-1.216, $\left.p=0.003\right)$

more accurately remember using the mobile phone at the time of the operation, especially in patients who underwent surgery in $90 \mathrm{~s}$ and the matched patients. Thus, based on our casecontrol study, we were not able to conclude that mobile phone use increased tumor incidence. These findings were similar to those of previous case-controlled studies [4-7]. Furthermore, those studies had various uncontrollable factors and limitations in reliability $[5,19]$, thus questions regarding their findings would have arisen even if mobile phone use had been found to increase tumor incidence.

A case-case study was conducted only in the patient group, and tumor volume was found to be clearly larger for the regular user group compared to the nonuser group. In addition, tumor volume was significantly larger in both the daily and cumulative heavy user groups compared with the light user group amongst regular cell phone users. Previous reports showed similar results as those found in our study. A Danish study [4] reported that the mean size of vestibular schwannomas was significantly larger in regular mobile phone users compared to nonusers, with $1.66 \mathrm{~cm}^{3}$ in users and $1.39 \mathrm{~cm}^{3}$ in nonusers. An increased risk for schwannomas was observed in regular mobile phone users compared to nonusers, and was also seen in patients who reported having used mobile phones at the affected ear for $>20 \mathrm{~min} /$ day on average. In that study, two possible explanations were suggested for these results. One was that the increased risk was caused by exposure to the EMFs from the mobile phone, and the other was that the higher risk came from selection bias and/or recall bias. A selection bias might distort the results if heavy users with ipsilateral mobile phone use were more likely to participate in the study because of the earlier detection of tumors than those in the general population.

According to Inskip's assumption, there was possible risk from mobile phone only when the ear used most frequently for speaking on mobile phones and the tumor location were ipsilateral. In previous studies, the odds ratio for the more frequently used ear was significantly higher among long-term users (1.8 to 3.9 of odds ratio) when analyses took into account the ear used during mobile phone use and the side at which the tumor developed [11, 22]. On the other hand, other studies have reported that the odds ratio for the more frequently used ear was not significantly higher (0.82 to 1.08$)[24,25]$. However, previous studies have not considered the patient's hearing. In cases where a patient had lost his or her hearing due to the development or growth of a tumor, the patient's preferred ear for mobile phone use may have changed due to the hearing loss. Therefore, bias needs regarding the correlation between the frequently used ear and the location of the tumor development to be reduced. In our study, we accounted for the patients' hearing in our analysis in order to reduce the

Table 4 Case-only analysis of regular users. A comparison of tumor volume according to duration, daily usage time, and cumulative hours of mobile phone use

\begin{tabular}{|c|c|c|c|c|}
\hline & Tumor size $\left(\mathrm{cm}^{3}\right)$ & & Odds ratio $(95 \% \mathrm{CI})$ & $p$ value \\
\hline Duration $(\leq$ or $>10$ years $)$ & $\begin{array}{l}\text { Long-term user }(n=38) \\
9.83 \pm 11.97\end{array}$ & $\begin{array}{l}\text { Short-term user }(n=26) \\
5.57 \pm 8.15\end{array}$ & $1.045(0.987-1.107)$ & 0.130 \\
\hline Time ( $\leq$ or $>20 \mathrm{~min} /$ day $)$ & $\begin{array}{l}\text { Heavy user }(n=32) \\
11.32 \pm 15.43\end{array}$ & $\begin{array}{l}\text { Light user }(n=32) \\
4.88 \pm 5.60\end{array}$ & $1.073(1.008-1.141)$ & 0.026 \\
\hline Cumulative hours $(\leq$ or $>2,000 \mathrm{~h})$ & $\begin{array}{l}\text { Heavy user }(n=26) \\
13.31 \pm 14.07\end{array}$ & $\begin{array}{l}\text { Light user }(n=38) \\
4.88 \pm 6.16\end{array}$ & $1.088(1.023-1.157)$ & 0.007 \\
\hline
\end{tabular}


Table 5 A regular user case-only analysis. Tumor side and mobile phone use

\begin{tabular}{|c|c|c|c|c|c|c|c|}
\hline & \multirow[t]{2}{*}{ Tumor side } & \multicolumn{3}{|c|}{ Regular side for phone use } & \multirow[t]{2}{*}{ Total } & \multirow{2}{*}{$\begin{array}{l}\text { Odds ratio } \\
(95 \% \mathrm{CI})\end{array}$} & \multirow[t]{2}{*}{$p$ value } \\
\hline & & Right & Left & Both & & & \\
\hline \multirow[t]{3}{*}{ Patients in all hearing levels } & Right & 12 & 20 & 5 & 37 & \multirow[t]{3}{*}{$0.733(0.236-2.282)$} & \multirow[t]{3}{*}{0.592} \\
\hline & Left & 9 & 11 & 7 & 27 & & \\
\hline & Total & 21 & 31 & 12 & 64 & & \\
\hline \multirow[t]{3}{*}{ Patients limited to serviceable hearing } & Right & 7 & 3 & 2 & 12 & \multirow[t]{3}{*}{$4.500(0.585-34.608)$} & \multirow[t]{3}{*}{0.148} \\
\hline & Left & 3 & 5 & 1 & 9 & & \\
\hline & Total & 10 & 8 & 3 & 21 & & \\
\hline
\end{tabular}

bias regarding change in hearing. Furthermore, for regular mobile phone users, we reclassified the frequently used ear based on the transition period, where the patient changed their mobile phone use from the ear on the lesion side to the other ear due to hearing loss. However, since bias may arise in the reclassifying process, data were reassessed only in those patients who had serviceable hearing; the results showed that the lesion side that developed a tumor and the frequently used ear had a high correlation with each other (Table 5). This correlation may have significant meaning and meet the Inskip's assumption, as patients who did not use a mobile phone had a similar tumor incidence for both sides (Table 3 ).

Takebayashi et al. [6] examined the tumor diameter in cases with ipsilateral mobile phone use versus cases with contralateral mobile phone use and reported that the diameter of ipsilateral-side tumors was smaller. The reason for these results was thought to be that cases with ipsilateral use were more likely to be diagnosed at an earlier stage than cases with contralateral use. At the same time, it is possible that ipsilateral tumors may grow more slowly. However, there was no significant difference in tumor volume between ipsilateral and contralateral users in our study.

We conclude that there was a consistent association between tumors and mobile phone use, with a greater relationship to tumor growth than incidence. We suggest that local heating caused by mobile phone use may result in a thermal effect, which may promote growth of an already existing schwannoma. At the same time, the effect of energy absorption at tissue sites close to the mobile phone needs to be clarified $[11,15]$. If the energy from mobile phone use can cause tissue degeneration at the protein level, then there is also the possibility that these waves may induce changes in tumor growth and characteristics [12, 13, 26, 27].

Our study had some limitations because of the previously mentioned biases, but considering that prospective study of this particular topic would be very difficult to conduct, we believe that these biases are within an acceptable range. Many efforts were taken to reduce bias in the design of our study, especially in the case-control study with prevalent controls. We selected our controls among the subjects who received comprehensive routine medical checkups including brain MRI.

There are differences in the absorption of electromagnetic waves in the brains of adults and children, but these differences were not evaluated in our study. However, vestibular schwannoma occurs mainly in people aged 50 years or older, with the exception of type II neurofibromatosis which was not included in our study [28]. In this study, all patients except one were adults.

Another limitation of this study was that recall bias, residency, age, EMF according to cell phone type, and use of other electronic devices were not considered. In order to obtain accurate statistical data, other factors, such as residency, duration of use of each electronic device, use of microwave ovens, computers, televisions, amateur radios, Bluetooth devices, and cordless phones in the home need to be thoroughly evaluated. Realistically, however, it is difficult for participants to remember accurately and it is nearly impossible for researchers to control all these factors. In addition, a recall bias may develop if questions are asked repeatedly in order to gather more information. As all other factors were equal, we predicted that there would be no statistical differences between the two groups. Under this supposition, there was no significant relation between mobile phone use and tumor incidence, whereas a significant change was observed in tumor volume and mobile phone use in our study. Therefore, we predict that mobile phone use and tumor growth are correlated with each other. So, if patients were diagnosed with vestibular schwannoma, we should advise them to refrain from mobile phone use.

\section{Conclusion}

From our results, we found that tumors tended to coincide in the more frequently used ear when talking on mobile phones, and tumor volume showed strong correlation with the amount of mobile phone use, thus there is a possibility that mobile phone use may affect existing tumors growth. 
Acknowledgement This study was supported by a grant from the National Research Foundation of Korea (Project number 2012R1A1A2004323).

Financial disclosure There is no financial relationship with any commercials. I have nothing to disclose about financial support.

\section{Conflict of interest None}

Open Access This article is distributed under the terms of the Creative Commons Attribution License which permits any use, distribution, and reproduction in any medium, provided the original author(s) and the source are credited.

\section{References}

1. Lanser MJ, Sussman SA, Frazer K. Epidemiology, pathogenesis, and genetics of acoustic tumors. Otolaryngol Clin North Am. 1992;25(3):499-520.

2. Howitz MF et al. Incidence of vestibular schwannoma in Denmark, 1977-1995. Am J Otol. 2000;21(5):690-4.

3. Lai H, Singh NP. Acute low-intensity microwave exposure increases DNA single-strand breaks in rat brain cells. Bioelectromagnetics. 1995;16(3):207-10.

4. Christensen $\mathrm{HC}$ et al. Cellular telephone use and risk of acoustic neuroma. Am J Epidemiol. 2004;159(3):277-83.

5. Ahlbom A et al. Epidemiologic evidence on mobile phones and tumor risk: a review. Epidemiology. 2009;20(5):639-52.

6. Takebayashi $\mathrm{T}$ et al. Mobile phone use and acoustic neuroma risk in Japan. Occup Environ Med. 2006;63(12):802-7.

7. Muscat JE et al. Handheld cellular telephones and risk of acoustic neuroma. Neurology. 2002;58(8):1304-6.

8. Repacholi MH. Health risks from the use of mobile phones. Toxicol Lett. 2001;120(1-3):323-31.

9. Moulder JE et al. Mobile phones, mobile phone base stations, and cancer: a review. Int J Radiat Biol. 2005;81(3):189-203.

10. Lonn $\mathrm{S}$ et al. Mobile phone use and the risk of acoustic neuroma. Epidemiology. 2004;15(6):653-9.

11. Schoemaker MJ et al. Mobile phone use and risk of acoustic neuroma: results of the interphone case-control study in five North European countries. Br J Cancer. 2005;93(7):842-8.
12. Johansen $C$ et al. Cellular telephones and cancer: a nationwide cohort study in Denmark. J Natl Cancer Inst. 2001;93(3):203-7.

13. Repacholi MH. Radiofrequency field exposure and cancer: what do the laboratory studies suggest? Environ Health Perspect. 1997;105 Suppl 6:1565-8.

14. Foster KR, Glaser R. Thermal mechanisms of interaction of radiofrequency energy with biological systems with relevance to exposure guidelines. Health Phys. 2007;92(6):609-20.

15. Baan $\mathrm{R}$ et al. Carcinogenicity of radiofrequency electromagnetic fields. Lancet Oncol. 2011;12(7):624-6.

16. INTERPHONE Study Group. Acoustic neuroma risk in relation to mobile telephone use: results of the INTERPHONE international case-control study. Cancer Epidemiol. 2011;35((5):453-64.

17. Lu YS, Huang BT, Huang YX. Reactive oxygen species formation and apoptosis in human peripheral blood mononuclear cell induced by $900 \mathrm{MHz}$ mobile phone radiation. Oxid Med Cell Longev. 2012;2012:740280.

18. Liu $\mathrm{C}$ et al. Exposure to $1,800 \mathrm{MHz}$ radiofrequency electromagnetic radiation induces oxidative DNA base damage in a mouse spermatocyte-derived cell line. Toxicol Lett. 2013;218(1):2-9.

19. Schuz J. Lost in laterality: interpreting "preferred side of the head during mobile phone use and risk of brain tumor" associations. Scand J Public Health. 2009;37(6):664-7.

20. Hartikka $\mathrm{H}$ et al. Mobile phone use and location of glioma: a casecase analysis. Bioelectromagnetics. 2009;30(3):176-82.

21. Inskip PD et al. Cellular-telephone use and brain tumors. N Engl J Med. 2001;344(2):79-86.

22. Lonn $\mathrm{S}$ et al. Incidence trends of adult primary intracerebral tumors in four Nordic countries. Int J Cancer. 2004;108(3):450-5.

23. Cardis $\mathrm{E}$ et al. The INTERPHONE study: design, epidemiological methods, and description of the study population. Eur J Epidemiol. 2007;22(9):647-64.

24. Sato $\mathrm{Y}$ et al. A case-case study of mobile phone use and acoustic neuroma risk in Japan. Bioelectromagnetics. 2011;32(2):85-93.

25. Larjavaara $\mathrm{S}$ et al. Location of gliomas in relation to mobile telephone use: a case-case and case-specular analysis. Am J Epidemiol. 2011;174(1):2-11.

26. Hardell L et al. Cellular and cordless telephones and the risk for brain tumors. Eur J Cancer Prev. 2002;11(4):377-86.

27. Auvinen $\mathrm{M}$ et al. Ornithine decarboxylase activity is critical for cell transformation. Nature. 1992;360(6402):355-8.

28. Stangerup SE, Caye-Thomasen P. Epidemiology and natural history of vestibular schwannomas. Otolaryngol Clin North Am. 2012;45(2):257-68. vii. 